\title{
基 調講演
}

\section{Nursing Phenomena and Research Strategies}

\author{
From Nurse's Concern to Researchable Question
}

\author{
Pamela J. Brink, RN, PhD, FAAN \\ Professor Emeritus \\ University of Alberta
}

\section{Nursing research is a journey of exploration that begins with a question}

Nursing research is a journey of discovery. It is a process or a disciplined movement from the unknown and the uncertain to the known and the reliable. Because it is nursing research it involves knowledge that is of use to nursing in all its practices-whether education, administration, research or clinical practice. Nursing research is interested in the basic and social sciences that underpin our practice; interested in applied sciences that feed into our practice; and, interested in the specific application of information to our patient populations and our own behavior. We want to know all about our patients, from all perspectives. We want to know all there is to know about our own practice: its flaws and how to improve it. We want to know from the molecular level to the interactive behavioral level. We are eclectic. We are specialists and we are generalists. In the end, it is the need to know how to improve our care that is paramount.

All research begins with a question. Answering questions has always been the special task of the researcher. Knowledge is only built through the answers to very specific questions. Broad, diffuse, rambling, inarticulate questions are of little use to a researcher, but sound, clear, directive, precise questions are critical to the success of any research project.

What phenomena are of interest to nurses? Just about everything. Nursing is an applied discipline that focuses on the human being who is ill or disabled, may become ill or disabled, or is recovering from an illness or trying to cope or live with an illness or disability. Nursing is interested in people of all ages, cultures and races. Nursing is interested in people in the home, in the hospital, and in the community. Nursing is interested in human organization, the individual person, families, kin groups and towns. Nursing is interested in educating other nurses as well as educating patients, former patients or potential patients.

The breadth of information of interest to nursing is overwhelming and at the same time it allows many different kinds of research questions. Since no single piece of research can possibly answer all the questions nurses need to have answered, each of us is responsible for finding an area of research of interest to us and pursuing that interest for a lifetime. Even then, we will not have exhausted the possible questions about our topic of interest.

\section{All research is on some topic of phenomena}

The first task of a researcher, then, is to decide what to study. Since the possibilities are almost endless, the matter of choosing only one phenomenon can be intimidating. Research topics come from experience and background. Generally a research topic is something of 
personal interest.

My advice to any new researcher is to take some time to meditate on one's own life. What seems to be the major thread or threads of interest to you? When you go to a bookstore to buy a book, what topics are you most likely to buy to read? When you were in nursing school, which classes most interested you? Which ones drew you to study more often or more intensely? Which clinical experience fascinated you the most? Not all were equally stimulating - which one drew you most personally? And what about your own life experience? Have you, a family member or a friend, experienced an illness or trauma that affected you deeply and personally?

All of these areas can provide you with an indication of a personal interest in a particular phenomenon that will sustain your interest for a lifetime. If you are not personally interested in the phenomenon, then your research will be dry, dull, uninteresting and probably uninformative. In addition, you will do no further research beyond the study required for your degree. If, however, you are deeply interested in your topic, you will never stop asking questions that need to be solved.

\section{Next, discover what you know about the topic}

Consider what you know about your topic and how you know about it. Is your knowledge based solely on personal experience? Have you done any reading or exploration about your topic? You can make a list with two columns : what I know; what I don't know. (You can see, at this point, that if you are not interested in the topic you are already bored with it.) From this exploration, you can make a new list headed, "What I want to know about my topic."

Once you know the direction you want to take and the gaps in your information, go to the literature to see if your question ("What do I want to know about this topic?") has already been answered. If the question has not been answered to your satisfaction, you can now become more directive in your literature search.

The research literature on your general area of interest needs to be examined from very specific perspectives :
1. What is the literature base on your phenomena? When you do a CINAHL or Medline search, how many articles will your search term elicit?

2. You can categorize the research into methods of data collection or research designs.

3. You can categorize the research by the theory or conceptual base for the research. Do other researchers have the same reason for exploring these phenomena as you?

4. You can categorize the research by country of origin. Has anyone done this research in your country or on your people?

5. Are the research subjects used in these studies of interest to you or has the literature missed your population of interest?

\section{The research literature tells you the state of knowledge about your topic}

From this initial categorization of the literature base, you should get some sense of the state of knowledge about your topic. You can arrange the publications by date of publication. How long have researchers been working in this area? The longer the time span is, the greater the knowledge base becomes.

Now you know what research has been completed on your topic and what answers the previous researchers found. Since these computerized data bases usually have an abstract of the article for you to read, you can find even more information about your topic and what is known. Only after this very thorough search will you know whether or not your very general question on your selected phenomena has been answered by another researcher.

The literature search not only provides you with the most current knowledge about your phenomena, it also provides you with clues as to what is missing from the data. The knowledge gaps are identified only after the literature has been examined over and over again. Remember that research means "to search and search again." This also applies to the initial literature review.

You may have found by this time that the more specific your question, the easier it is to do a literature search. You may want to focus your search on a particu- 
lar population or procedure for care. If, for example, you are interested in diabetic patients, what is it about them that you want to know? Do you want to know how to improve compliance with the diabetic treatment regimen? Do you want to know the best methods of teaching them about their disease? Do you want to know how to prevent complications? Do you want to know which ethnic groups have a higher incidence of diabetes and which type? Do you want to know about the age of onset? Just what is it that you want to know?

There is an enormous literature base on diabetes and diabetic patients. Your literature search must become narrower and narrower and more and more specific as you discover just how large the data base is. This, in itself, will give you an indication of just what kind of research you will be doing on your topic.

If, on the other hand, there is very little literature on your topic-no matter how hard or how long you have searched-then your question will remain broad and exploratory. The smaller the literature base, the broader the research question, the larger the literature base, the narrower and more specific the research question.

Finally, the literature will let you know whether or not the nursing profession is even interested in your topic. If no nurse or nursing journal has ever published on these phenomena, there may be a reason for it. The topic may be of interest to another discipline but not nursing. Look where the literature has been published, if any.

If you do find your topic or phenomena published in nursing journals, what do other nurses have to say about why it is important to nursing? You can find the answer to this question in the beginning of the article listed under "literature review" or "the research problem" or the general argument provided for why this research is important. You should not have to read a special section called "Significance for Nursing" if the author has done a proper job and explained why the research is important and why it must be done.

This section of a research report is very important to read as it provides you with how the authors "grounded" their research in the current literature. How did they explain where they got their idea? How did they explain why they chose the research design, the method of data collection and analysis, the population for study? All this lets you know the significance of this research for the discipline of nursing. From your reading of the literature, you are now ready to decide what kind of research you want to do on your topic.

\section{Research questions lead to two basic paradigms}

When Marilynn Wood and I wrote the fist edition of Basic Steps in Planning Nursing Research: From Question to Proposal, we were only dealing with the scientific paradigm. At the time, we felt that qualitative research designs - which were just being formulated and written about-belonged at the first level of research: the indepth exploration of a single topic or concept. The first level of research design, therefore, included all of the qualitative research designs. These we entitled "Exploratory" designs as they did indeed "explore" a single broad topic in depth.

Today, what we included under exploratory designs have now been developed as a corollary research process to the Scientific Paradigm and labeled the "Naturalistic Paradigm" (See Figure). The emphasis in the two paradigms differs. Within the scientific paradigm, the emphasis is upon the building of knowledge from small exploratory single investigations to large correlation designs leading to theory to explain the findings of the interrelationships between and among variables. From these tentative theories, comparative and experimental designs were created to test those theories.

At this point, you need to decide whether you will be doing research within the scientific paradigm or the naturalistic paradigm. You cannot do both at the same time within the same project. They are fundamentally different in their philosophies as we shall see.

\section{The scientific paradigm and the naturalistic paradigm}

The underlying philosophy of the scientific paradigm is that no single research study can completely answer the question. Research in this paradigm builds from the exploratory or pilot study to the highly 


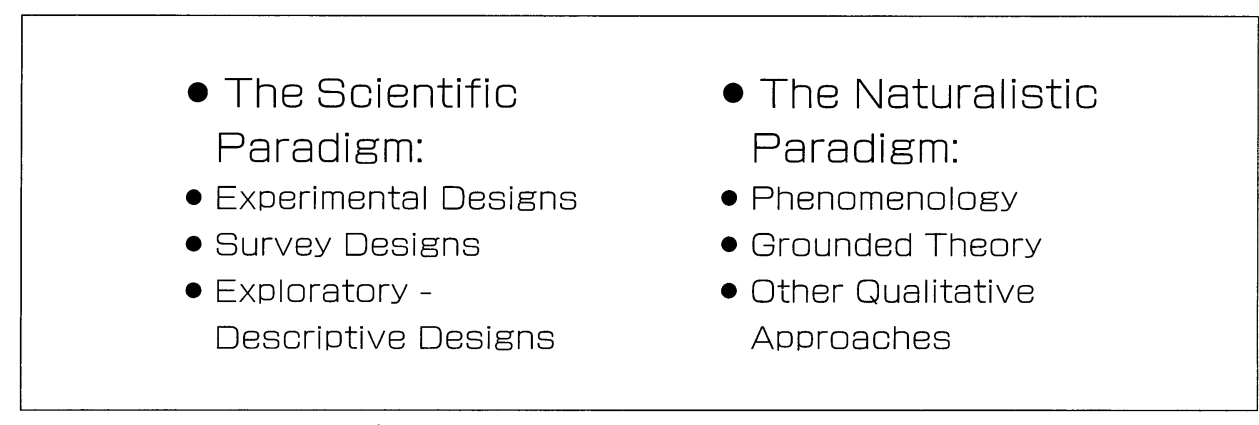

Figure

Research questions lead to two basic paradigms

controlled experimental design identified in the literature review, As I said, within the scientific paradigm, the work builds from exploratory-descriptive designs to theory testing designs in the form of comparative or experimental designs. The greater the literature base, the more likely the knowledge base will lend itself to the experimental design. This is where we, as nurses, test our clinical practice.

Within the naturalistic paradigm, the more research on the topic, the more likely someone else has already answered your question and you must ask another. The underlying philosophy of the naturalistic paradigm is that each study is both a beginning and an end in itself. There is no "building" of knowledge from study to study but rather an intense in-depth exploration of a single question within one study.

I will not be talking about the naturalistic paradigm today since each design has different philosophies and goals. These studies normally have very small sample sizes, have very little literature base on the specific topic to be studied (otherwise an intensive in-depth study would not be undertaken), and have very specific outcomes.

Phenomenology is philosophy driven in which the specific philosophy underpinning the research must be adhered to faithfully throughout the research process. The process of conducting a phenomenological research study differs drastically from all other research studies and the methods and terminology used for these studies are not appropriate for others. Phenomenology does not intend to generate theory, instead it's intent is to fully explore a particular human condition in order to share with others the meaning and significance of the event.

Grounded theory, on the other hand, is an in-depth exploration of a topic designed to generate theory about the findings. Once again, the methods of sample selection, data collection with concurrent analysis, validation and paper trails are not suitable for other research designs.

Ethnographic research is often listed within the naturalistic paradigm and is so in the sense that the field setting for the research is uncontrolled, the sample sizes are small, the research is in-depth rather than in-breadth, and in depth interviews (both formal and informal) are one arm of the data collection process. It comes under the scientific paradigm in that it also builds, over time, from small exploratory studies to survey designs and finally even to field experiments on the same population. It uses a minimum of three methods of data collection at the first or exploratory phase and always usesparticipant observation coupled with informal and formal interviews. Other methods of data collection include artifacts of daily living (present and historic) photography (stills and movies), written documents and so on. Triangulation of data collection methods, triangulation of informants, and longitudinal research are all hallmarks of this design.

Embedded within every question is the nucleus of its answer. Research questions, like very other question, direct the answer. Therefore, the answer to the research question is determined by the question itself. 


\section{The answer is in the question}

"The answer is in the question" is an old Chinese proverb imminently suited to research. From this brief saying, Marilynn Wood and I created the research text Basic Steps in Planning Nursing Research: From Ques. tion to Proposal first published in 1978 . We believed that not only could research be directed by a question, but a research text could also be directed by the questions asked.

The structure of a question, in the English language, directs the listener to answer the question in a particular way. Children learn from earliest infancy which questions are rhetorical, which questions require an answer, and which questions are not questions at all. The questions require a particular kind of answer depending upon the question asked.

If a parent asked a child, "Why did you do that?" the child immediately knows an explanation is called for. Sometimes a child responds, "I didn't do it, it was the cat!" thereby attempting to distract the parent by laying blame elsewhere. Or the child may simply respond, "It was an accident" which is an explanation. Younger children may say, "I don't know."

Other questions may be similar to "Who is that?" which means "tell me all about that person." "Where are you going?” requires the child to specify a place, companions, "Who with?" and probably also some time element, "When do you plan to come home?"

Questions that begin with "how" are often process questions. "How did you do that?" Or "How does that work?" How questions are exceptionally hard to formulate into an appropriate research design and we recommend they be rewritten into a question that begins with "what." In fact, we recommend that all questions that do not begin with what be rewritten so that they do.

\section{The first word of the research question is critical}

We believe, that if a question is structured in a particular way, the answer to the question is easier to formulate. We initially recommend, therefore, that the question begin with the questions: who, what, where, when, how and why.

I do not know the structure of the Japanese language and I don't know if the structure of English questions translates into Japanese conceptual frameworks. My conceptualization of the research process is limited and restricted by my language.

Newspaper journalists are always required to answer these basic questions within an article they write in order to completely describe an event. Every English speaking child learns that these are basic life questions that must be answered. We believe, therefore, that if the research question begins with these words, they set the general tone for the rest of the research question. No other word or phrase is allowed to precede these words. If, as you practice writing your research questions, and you find other words creeping in before these words, rewrite the question until these words come first!

The first word of a research question tells you whether the answer is going to be a description or an explanation. In the first edition of our text, published in 1978, we included all the words - who, when, where and how-but we discovered over the years and countless students and research courses, that we need only use "what" as our first word. Who, where, and when are all part of the description of the research plan. "What" always leads to description. The first words to a "what" question then become either "What is......." or "What are......"

\section{Descriptive studies generally begin with a "what" question}

You will notice that the question begins with what and follows with one variable and one sample. This is the standard structure of a first level of a "what" question. The beginning exploration and description of a phenomenon about which little is known either about the topic or about the population. In addition, I focus upon the structure of questions, rather than the content of the topic or phenomenon.

- What are the health beliefs of the Ainu?

- What are the decision making strategies of families with a member who has recently been diagnosed with cancer? 
What are the health beliefs of the Ainu? The very broad topic is "health beliefs" and the population is "Ainu". What are the decision making strategies of families with a member who has recently been diagnosed with cancer? The broad topic is "decision making strategies" and the population is "families with a member who has recently been diagnosed with cancer".

These are very broad, very exploratory questions that assume there is little literature on the topic. If, after you have reviewed the literature on these topics, you discover there has been a great deal of research on either the topic or the population, you will need to rewrite your question into something that is either more specific or at another level of research design.

- What are the characteristics of persons with anorexia nervosa?

The broad topic is "characteristics" (which means any kind of demographic characteristic as well as something more specific about the characteristics of the disease). The population is "persons with anorexia nervosa."

You will notice that I have shifted our thinking from "topic" or "phenomenon" to "variable." One of the things we learn from our literature review is just how our topic or phenomenon varies or changes under differing circumstances. Our research designs become directed by the type of variability in our phenomena. At the most beginning level of research (as well as later) variables are nominal-they are named variables with no numerical substance. All qualitative research deals with nominal variables to the exclusion of numerical (mathematical) variables.

As we learn more about our variables, they can be counted mathematically. We can ask, "How many of this, or how many of that?" We can ask, "Are there more of this than there is of that?" "Is there a progression?"

Remember that nominal variables are named variables that have no order. Ordinal variables are named variables that are ordered from lowest to highest or poor to excellent. Ordinal variables can also be numerical as in $1,2,3,4,5$. The notion of ordinal variables is the placement of the variable within the string. Metric variables are the mathematical variables which can be statistically manipulated to give meaning to the relationships among variables. One of our major concerns in our literature review is to know all there is to know all about the variability of our variable(s) in our study.

The following question has the same structure. What are the health care decision making strategies used by parents with sick children? "What" is the question; "Health care decision making strategies" is the variable, and "parents with a sick infant" is the population.

You will notice that I am speaking here only of the population-or the general description of the people/or objects/or whatever from which you will select your sample. How you go about selecting the sample from that population is the topic of yet another lecture.

The next question has the same general structure, but needs to be broken up a bit.

- What are the experiences of women during the first year of bereavement?

What" is the question, the topic is "bereavement experiences", and the population is "women during the first year of bereavement." The question could be rewritten as: What are the bereavement experiences of women during the first year of bereavement? Although this question appears awkward and unnecessarily repeats "bereavement", it is correctly written according to our rules.

\section{More complex "what" questions have more than one variable}

As the knowledge about a topic grows, more complicated questions can be developed. Notice, however, that the first word of the sentence is still "what" and the answer will still be a description. It is the topic that becomes more specific and narrower. In addition, the topic will contain two or more variables that can be counted in some way.

- What is the relationship between type of nutrition, level and type of exercise and level of blood cholesterol?

- What is the difference between obese and normal weight individuals in their cholesterol levels?

In this first question we are looking for a relationship between variables: nutrition, exercise and choles- 
terol levels. As you can see, the population has not been specified. We can select any population for study-we simply need to choose one that is large enough for us to be able to find all these variables, and hopefully all in single individuals in order to see if there is any relationship among them. The population must be large enough to see if there are any statistically significant relationships among all these types of variables. All are numeric, all will be statistically tested with some form of correlational analysis. This level of complexity will still yield only a description - albeit a statistical description. This question will lead to a correlational research design.

The second question is still a "what" question and will still yield a description for an answer. It is obviously a "next generation" question based upon the answer to the previous question which found differences in levels of cholesterol depending upon the weight of the individual. We are now going to construct a piece of research to test the theory that obesity is a "cause" of higher elevations of blood cholesterol. We do not know whether obesity indeed causes high cholesterol or if there is something else that causes both of them, but this question is still at the descriptive level testing a tentative theory. Obviously, we cannot create obesity in people in order to do this study, so we cannot do an experimental design. We can, however, select our sample in such a way that we will be comparing obese subjects with normal weight subjects on their cholesterol levels.

Notice that in the first question looking for the relationships among nutrition, exercise and cholesterol, a very large sample is needed to have any meaningful statistical correlations. You will also notice that in order to do this study, the variables need to be very precise and numerical. What do you mean by nutrition? Or exercise? Or cholesterol? Have you already categorized people into good nutrition and bad nutrition? If so, how did you do that? On what information? Or, on the basis of your literature review, did you decide to collect weekly food diaries from the sample, submit these diaries to a detailed nutritional analysis to determine the quality of certain foods such as the amount and type of fat ingested weekly, and compare that with the blood cholesterol levels obtained at the end of that week? To do this, you will need numerical variables to do the correlation. In addition, however, you have asked for the type and amount of exercise, which will also be found in the weekly food diary. These too will have been converted for use such as sedentary $=1$, moderate $=2$, athlete $=3$ (or some such scale). This variable will also be correlated with cholesterol levels and amount of fat.

It must be very obvious at this point that this question is based upon a great deal of previous research on each of the original variables which was found in the literature.

The second question, on the other hand, compares two specific groups of people on the basis of their weight those who are obese and those who are of "normal" weight. Based upon the literature review and your own research, you have discovered that obesity was indeed related to blood cholesterol levels. You then write a theory to explain why the obese have higher cholesterol levels than normal weight individuals. You will need to test your theory using only obese subjects and normal weight subjects. Your sample will need to be highly controlled so that both groups are identical on all demographic and health variables, with the single exception of their defined weight, however you have defined obesity and normal weight for your study.

Now you may be able to see the logic for the structure of a research question. All "what" questions, regardless of complexity, lead to descriptive research designs. The designs may be exploratory, purely descriptive, correlational or comparative-but these are all descriptive designs. The simplest "what" question has one sample and one variable. More complex "what" questions has one sample and multiple variables.

\section{"Why" questions have a different structure and process}

Why questions are entirely different from "what" questions. They are more complex requiring an intensive literature review on all variables as well as the population. Why questions are the culmination of all the research that has been done on "what" questions. Once we have a complete description of a phenomena and how it acts, responds and interacts, we can now ask why it 
does so.

Every "why" question is answered by "because......" Therefore, every "why" question demands an explanation for an answer. An explanation is another way of describing a theory.

Let's look at the following questions :

- Why does the ingestion of Omega three appear to reduce skin bruising in the elderly?

Can you answer this question? If you can, you know the literature and have the knowledge base to proceed. If you can't, you need to go to the literature to answer the question: What is the relationship between Omega 3 and epidermal fragility? If you can't find the answer in the literature, you must first answer the research question doing a correlational design. If, however, you find a physiological relationship that can trace skin fragility to a lack of Omega 3 in the blood, you may then use that literature to create a theory that explains why Omega 3, or the lack of it, influences bruising.

Just as a general aside, we have found that individuals who know a great deal about their topic before they begin to construct a research question usually have great difficulty constructing a simple one variable one sample "what" question. These individual may be able to write a why question and provide a theoretical answer without going to the library. In this case, these individuals should be able to trace the research from its earliest beginnings to its logical end point. Sometimes they must go back to the literature to construct the historical basis for their question.

- Why do formal classes on their disease process appear to increase adherence to the diabetic regimen in newly diagnosed diabetics?

Here again we have a "why" question that requires an explanation. Is it the formality of the classes or is it simply the educational process itself that increases adherence? The literature should give you the step by step process you need to create your theoretical explanation. The theory developed by the researcher to answer the "why" question is not mere speculation, it is a highly structured argument based upon the research literature.

The literature is reviewed on :

- The population to be studied.

- All of the research to date on both variables and how they relate to one another.

- Other populations experiencing the same variables.

From the literature review, the researcher constructs a step by step argument on how and why the variables influence each other. I am afraid I cannot go into detail today on how this is done. There are many examples of the rationale, conceptual framework, theoretical framework, or argument in the literature.

The scientific paradigm assumes that no piece single piece of research can answer the research question. This is even truer of theory. No single research study can support or defend a theory totally. As you develop hypothesis to test the theory, you will notice that hypotheses are very limited in nature and very precise-carving out only a small portion of the theory at a time.

\section{For the "why" question, it is the hypotheses and not the question}

The "why" question is significantly different from the "what" question in that it requires a theory base for the development of the research. From the research literature, a theory is constructed. From the theory an experiment is designed based upon predictive hypotheses.

A hypothesis is a statement of a specific relationship between two or more variables. At their simplest, hypothesis have two variables and one sample; an independent or causative variable; a dependent or result variable. The variables must be numeric in some form, whether nominal, ordinal or metric because the hypotheses are always tested statistically to determine whether or not there is a statistical significance in the outcome.

More complex hypothesis contain: one or more causal variables; one or more result variables; one or more intervening variables. As with all other progressions, any program of research will begin with simple predictive hypothesis for theory testing before going on the more complex hypothesis.

The structure of the hypothesis is the following. The first clause identifies the sample and one position of the independent variable. The second clause specifies the result or the dependent variable. The third clause specifies the other position of the independent variable. The 
structure of the hypothesis are following. The first clause identifies the sample and one position of the independent variable. The second clause specifies the result or the dependent variable. The third clause specifies the other position of the independent variable. Remember that the sample has already been defined and is very highly controlled in these studies. Depending upon the number of independent, intervening and dependent variables, one hypothesis is written for each predicted direction of change.

Examples of hypothesis are:

- Persons with frequent sub dermal skin bruising given 1,000 mg of Omega 3 daily for 3 months will have a significant reduction in bruising as compared to those who do not receive $1,000 \mathrm{mg}$ of Omega 3 daily.

- Persons newly diagnosed with diabetes receiving formal education on their disease will follow their diabetic diet more consistently than persons with informal teaching methods.

First hypothesis is the following. Independent variable : 1,000 mg Omega 3 daily for 3 months. Dependent variable: Amount of sub dermal skin bruising (metric variable). Population: Persons with frequent sub dermal skin bruising. Sample: Residents, including both men and women, at nursing home $\mathrm{A}$ who have been identified by the staff as having frequent skin bruising episodes.

Second hypothesis is the following. Independent variable: Formal educational classes on diabetes. Dependent variable: Adherence to dietary treatment regimen. Population: Persons newly diagnosed with diabetes. Sample : Males and females between the age of 40 to 60 years, newly diagnosed, attending clinic A who are literate (can read and understand the written materials on their disease)

Studies were done at one time looking to see "what will happen" if I do such and so. They are no longer approved by human ethics review committees. It is unethical to do an experimental design on humans based upon speculation. The horror of the human experimentation that occurred during World War II gave rise to this rule for researchers. Therefore, it is unethical to use an experimental design to deliberately cause harm to a human being.

\section{The comparative design can be used to test the hypothesis}

If there is a question about causing harm in an experimental design, the comparative design can be used to test the hypothesis as follows.

Population: Persons taking Omega 3 as a supplement versus those who do not. Sample: Hold age and ethnicity as a constant, comparable gender. Independent variable: Amount of Omega 3 found in the diet. Dependent Variable: Amount of sub dermal skin bruising.

Population: Persons newly diagnosed with diabetes receiving instruction about their disease (usual ward procedures). Sample: Comparable groups based upon age, gender and literacy. Independent variable : Type of instruction received, formal classes, one to one with nurse, handouts etc. Dependent variable: Adherence to dietary regimen over time.

The comparative design can be thought of as a "transitional" design. It is transitional because it is both theory testing and descriptive. Therefore it partially meets the criteria for both experimental designs (which are theory testing designs) and descriptive designs (which simply observe phenomena as they exist and do not manipulate or deliberately change them in any way).

We have found that many people have difficulty with the comparative design. Some feel that if the sample is deliberately selected on the independent variable in order to create two groups, that this is the same thing as manipulating the independent variable. That is not true. No one is deliberately changing the independent variable; the variable already exists in the population. Groups are formed by separating them on some dimension of the variable. The variable is not changed in any way. It is simply observed as it exists. The function of the comparative design is to determine if the outcome variable (dependent variable) differs significantly based upon the differences in the independent variable.

The comparative design is a transitional design because it not only tests theory, as in an experiment, it is also the next logical step in a program of research building upon the knowledge gained in a correlational design. Many correlational designs have large data bases at the end of a study and have interesting findings 
that can be explored further. The researcher may want to develop a theory to explain the findings but may not want to create an expensive experimental design to find out whether the theory works. In this case the researcher will design a comparative study, based upon that tentative theory.

Sometimes, the findings from a correlational study do not lend themselves to experimental manipulation. This is particularly true of nominal variables based upon some kind of personal demographic characteristic in the sample. We cannot change or manipulate a person's age, disease, or gender. We may find, however, that these characteristic variables have an impact upon our dependent variable.

Note the hypotheses in the following.

- Persons over the age of 60 will have significantly higher amounts of sub dermal bruising than persons under the age of 40 .

In this hypothesis, the independent variable is age. No matter what we do, we cannot make a person age for our study.

- Person with diabetes will have a higher incidence of infections than persons without diabetes.

In this hypothesis, it would be unethical to create in people in order to test our theory. We cannot manipulate age in an experiment but we can observe age by selecting the sample according to our age criteria. This creates the experimental design. We cannot ethically create infections in our diabetic patients in order to prove our point. We can substantiate our point through a literature review, or an epidemiological or comparative design.

\section{To review}

You may be wondering why I have spent so much time and attention on research questions that begin with "what." I believe that the state of nursing knowledge is such that we cannot ethically indulge in nursing intervention studies unless we have a pretty good idea what we are going to find. To do a nursing intervention study, we must have developed a theory, based upon previous research-our own or that of others-and we are testing that theory.

For the most part, we do not have the knowledge base in many areas of our practice to conduct ethical experiments. For this reason, I emphasize the type of research to develop that knowledge about ourselves, our patients and our health care systems. What works in one country or one culture group does not necessarily work with another. We need to find out why and to describe the differences. Not all patients respond the same way to their treatments or to their recovery process. We need to know what those differences are and whether they are unique to the individual or are common to certain groups.

Nurses are always involved with behavior - their own, their patient's, their student's, their colleagues. Human behavior is infinitely varied. Yet we must work with this variability on a daily basis. How can we know when we are effective and when we are not? Only through our research can we really know. Otherwise we simply speculate.

1. The simplest "what" questions (one sample and one variable) lead to exploratory designs. These studies frequently have little literature on the topic and are based upon small sample sizes. The variable in an exploratory study is usually a nominal variable. It may be very broad such as "characteristics", which is composed of a number of sub variables that can be nominal, ordinal or metric.

2. "What" questions also lead to descriptive designs that are based upon previous research on either the sample or the topic. In this case, the question is simply exploring the topic further.

3. More complex "what" questions (containing more than one variable) lead to correlational or comparative designs. Correlational designs are statistical designs requiring mathematical variables. They usually require very large samples which are statistically estimated by the size of the population. A clear description of the population characteristics is extremely important in these designs.

4. Correlational designs always look at relationships among two or more numerical variables and always require large samples. They are always based upon the findings from descriptive designs.

5. Comparative studies always look at "what is the difference between groups?" The sample is always 
divided into groups based upon one variable. They are always theory based. Comparative studies are transitional between correlational and experimental designs. They are both descriptive and theory testing.

6. Despite the complexity of correlational and comparative designs, they are still descriptive designs.

7. Experimental studies are created from "why" ques- tions. They are always hypothesis testing and always theory based and always the most highly controlled. Experimental designs are our nursing intervention studies and are only conducted when we have a very good theory to explain what will happen when we test an intervention. 